\title{
Isolated torsion of the fallopian tube associated with hydrosalpinx in a 17-year-old sexually inactive girl: an unusual case report
}

\author{
Angelos Daniilidis ${ }^{1}$, Sonia Haritidou ${ }^{1}$, STAMATIOS PETOUSIS ${ }^{1}$, Anastasios Liberis ${ }^{1}$, \\ CHRYSOULA MARGIOULA-SIARKOU ${ }^{1}$, and KONSTANTINOS DINAS ${ }^{1}$
}

${ }^{1}$ Aristotle University of Thessaloniki

January 10, 2021

\begin{abstract}
Isolated tubal torsion is a rare cause of acute abdominal pain in women and is even less frequent in the patients younger than 18 years. We present the interesting case report of a tubal torsion accompanied with hydrosaplinx, diagnosed in a 17-year old patient that was sexually inactive adolescent
\end{abstract}

Isolated torsion of the fallopian tube associated with hydrosalpinx in a 17-year-old sexually inactive girl: an unusual case report

Angelos Daniilidis, Sonia Haritidou, Stamatios Petousis, Anastasios Liberis, Chrysoula Margioula-Siarkou, Konstantinos Dinas

$2^{\text {nd }}$ Department of Obstetrics and Gynaecology, Aristotle University of Thessaloniki, Greece

\section{Abstract}

Isolated tubal torsion is a rare cause of acute abdominal pain in women and is even less frequent in the patients younger than 18 years. We present the interesting case report of a tubal torsion accompanied with hydrosaplinx, diagnosed in a 17-year old patient that was sexually inactive adolescent

\section{Keywords}

Obstetrics and gynaecology

Acute medicine

\section{Key Clinical Message'}

Isolated tubal torsion is an unusual cause of acute abdominal pain in young sexually inactive patients. However, it should be still taken into account regarding the differential diagnosis of such conditions.

\section{Introduction}

Hydrosalpinx is defined as a fallopian tube filled with fluid because of a complete distal occlusion and abnormal distension of the ampullary portion[1]. Isolated tubal torsion (ITT) is the rotation of the tube around its longitudinal axis, while the ovary and its blood flow remain unaffected [2]. Torsion of the right tube is more common than torsion of the left, as the left pelvic area is mainly characterized by the fixation of tube in the pelvic wall because of the sigmoid colon. ITT is a rare cause of acute abdominal pain in women and is even less frequent in the patients younger than 18 years[ 3,4$]$. 
Despite the fact that exact pathophysiology has not been yet outlined, there have been several factors hypothesized to contribute in this condition's appearance. Pelvic inflammatory disease, hydrosalpinx, endometriosis, paratubal, para-ovarian masses, hydatid cyst of Morgani, autonomic dysfunction of the fallopian tube, previous abdominal operations, history of peritonitis are some of the main causes of a tubal torsion [5-18]. However, relative conditions are mainly described in adults, while it is rather uncommon to diagnose ITT with hydrosaplinx in younger sexually non-active women.

We present the interesting case report of a tubal torsion accompanied with hydrosaplinx, diagnosed in a 17-year old patient that was sexually inactive adolescent with no previous abdominal surgeries.

\section{Case Presentation}

A 17-year-old girl was admitted as an emergency to our gynae department reporting nausea, over ten episodes of vomiting and convulsive pain to the left lower abdomen. Symptoms initiated 2 hours before admission to hospital. She had irregular menstrual cycle, and a previously normal gynecological assessment 2 years ago. She had no history of sexual intercourses and also no history of any gynecological or any other abdominal surgery. On admission, blood pressure was $135 / 75 \mathrm{mmHg}$, pulse $90 / \mathrm{min}$, body temperature was $36^{\circ} \mathrm{C}$ and oxygenated hemoglobin was normal. She had a BMI of 23.

Clinical examination revealed normal bowel movements on auscultation, but also tense and sensitivity on abdominal palpation mainly to the left lower quadrant. No vaginal examination was performed, as the patient was virgo and there was no sign of vaginal bleeding. Peripheral blood test showed mild leukocytosis $11.100 / \mu \mathrm{L}$ and normal C-reactive protein of $1.3 \mathrm{mg} / \mathrm{L}$. The hemoglobin level was of $11.7 \mathrm{~g} / \mathrm{dL}$. Transabdominal ultrasound demonstrated a large unilocular cyst of about $8 \mathrm{~cm}$ in diameter in the left ovary with reduced vascularity. Based on clinical and ultrasound findings, there was a high suspicion of ovarian torsion and a computed tomography (CT) was asked in order to confirm or exclude the potential diagnosis of ovarian torsion. Decision for surgical treatment with laparoscopy was thereafter decided. (Fig.1)

Laparoscopy was performed under general anesthesia. Four trocars were used: one of $12 \mathrm{~mm}$ diameter at the umbilicus and three peripheral of $5 \mathrm{~mm}$ to the lower abdomen. Intraoperative findings included a massively enlarged fimbrial funnel and a paraorian cystic tumor with torsion of the distal part of the fallopian tube (Fig.2). The tumor appeared infarcted, while inflammatory fluid was released after puncture. Puncturing the hydrosalpinx and completing a detorsion did not improve the aspect of necrosis. The other ovary was macroscopically normal as well as the rest of the abdominal cavity. No signs of possible infectious disease were identified.

In order to preserve ovary, the necrotic mass was resected at the level of corresponding mesosalpinx from the proximal to the distal end of the fallopian tube, therefore performing a left salpingectomy. The cystic tumor was later sent for pathological examination, which indicated acute hemorrhagic necrosis as well as the presence of a hydrosalpinx were described.

The patient recovered from the surgery without any complications and was discharged uneventfully the first postoperative day.

\section{Discussion}

This case report describes the extremely rare clinical entity of an isolated tubal torsion with coexisting hydrosaplinx in a17-year-old patient without history of sexual intercourses.

A possible explanation for ITTH in adolescents could be the presence of a congenital malformation of the tube in the peripubertal period. As the reproductive axis is stimulated between 9 and 14 years, menses may activate ovarian and tubal function, revealing a previously asymptomatic distal occlusion of the tube. An episode of asymptomatic pelvic inflammation near tubes may cause a distal occlusion, hydrosalpinx and then torsion. Besides, torsion of the hydatid cyst of Morgani, located near the fimbriated end of the tubes, could also cause the pathologic process. 
Diagnosis of ITT is usually difficult because symptoms are non-specific and common with many other conditions [6]. The typical presentation of ITTH is acute lower abdominal pain with nausea and vomiting, but no specific clinical feature allow with safety to distinguish this from torsion involving the whole adnexa. Absence of fever and normal C-reactive protein levels may be helpful to make the differential diagnosis from appendicitis.

Regarding most common location, Boukaidi et al.(2011) conducted a review of the literature and targeted reports published from 1999 to 2009 where 13 cases of ITTH in adolescents were reported[2]. In their series ITTH occurred on the left side in 9 of the 13 cases. This might suggest that ITTH occurs more frequently on the left tube although confirmation by a larger series of patients is needed.

Ultrasound is the imaging modality of choice as it is non-invasive and avoids radiation exposure but diagnosis is not always definitive. Abdominal ultrasound showing the fallopian tubes as fluid-filled tubular structures folded onto themselves to form a C or S hape and separated from ovaries is consistent with a diagnosis of hydrosalpinx. Color Doppler may be useful, but the presence of normal flow does not necessarily rule out torsion [19-21]. Computerized tomography (CT) as well as magnetic resonance imaging (MRI) are of some value as they may indicate a thickened fallopian tube, twisting of the adnexal pedicle, eccentric thickening and a septal appearance of the fallopian tube dilated and fluid-filled[19-21]. However, the gold standard for confirming diagnosis is laparoscopy, with all relative advantages of minimally invasive procedure that permit quick recovery and minimal morbidity [22].

Finally, severity of the disease are significantly affected by duration and extent of torsion. Boukkaidi et al proposed a classification of the tubal status by conducting salpingoscopy. Grades I and II would correspond to potentially salvageable fallopian tube, whereas grades III or more would require salpingectomy. According to this proposal, Grade I and II are treated by puncturing the hydrosalpinx and completing a detorsion. The correction of the distal occlusion is established by salpingoplasty few weeks later. In contrary, grade III represents a compromised tube that indicates the necessity of salpingectomy [2].

In conclusion, isolated tubal torsion associated with hydrosalpinx in children and sexually inactive adolescents is an extremely rare entity. Its presentation raises difficulties in the differential diagnosis. Ultrasonography with Doppler should be the first-choice imaging approach, but laparoscopy is the gold standard of diagnosis and therapy.

\section{Conflict of interest}

Authors declare no conflict of interest

\section{Ethical approval}

No ethical approval was necessary for the present case report according to our University legislation. However, informed consent was taken from the patient.

\section{Funding information}

No finding was received for the present manuscript

\section{Acknowledgements}

Authors have no other person to acknowledge for the present manuscript.

\section{Author Contribution'}

$\mathrm{AD}, \mathrm{SH}$ and SP conceived the idea for the present manuscript. $\mathrm{AD}, \mathrm{SH}, \mathrm{SP}$ and $\mathrm{C}$ M-S wrote the initial draft. AL and KD critically reviewed the initial draft. All authors have contributed significantly in the authorship of the present manuscript.

\section{References}


[1] Ng KYB, Cheong Y. Hydrosalpinx - Salpingostomy, salpingectomy or tubal occlusion. Best Pract Res Clin Obstet Gynaecol. 2019

[2] Boukaidi SA, Delotte J, Steyaert H, Valla JS, Sattonet C, Bouaziz J, Bongain A. Thirteen cases of isolated tubal torsions associated with hydrosalpinx in children and adolescents, proposal for conservative management: retrospective review and literature survey. J Pediatr Surg. 2011

[3] Gaied F, Emil S, Lo A, Baird R, Laberge JM. Laparoscopic treatment of isolated salpingeal torsion in children: case series and a 20-year review of the literature. J Laparoendosc Adv Surg Tech A. 2012 Nov;22(9):941-7.

[4] Demirel BD, Hancioglu S, Bicakci U, Ariturk E, Bernay F. Isolated tubal torsion: A rare cause of acute abdomen in childhood. Pediatr Rep. 2018 Apr 3;10(1):7604.

[5] Noviello C, Romano M, Papparella A, Ciavattini A, Martino A, Cobellis G. The isolated tubal torsion: an insidious pediatric and adolescent pelvic urgency. Pediatr Med Chir. 2018 Dec 4;40(2)

[6] Webster KW, Scott SM, Huguelet PS. Clinical Predictors of Isolated Tubal Torsion: A Case Series. J Pediatr Adolesc Gynecol. 2017 Oct;30(5):578-581.

[7] Wang CJ, Go J, Liu YC. Isolated Tubal Torsion With Endometriosis. J Minim Invasive Gynecol. 2017 May-Jun;24(4):512-513.

[8] Sozen I, Kadako R, Fleischman S, Arici A. Diagnosis and laparoscopic management of a fallopian tube torsion following Irving tubal sterilization: a case report. Surg Endosc. 2002 Jan;16(1):217.

[9] Feng L, Liu Y, Liu H, Chen X. Chronic isolated fallopian tube torsion associated with huge hydrosalpinx and hemosalpinx in a postmenopausal woman: a case report and brief review. Eur J Obstet Gynecol Reprod Biol. 2012 Oct;164(2):235-6

[10] Kondo E, Tabata T, Shiozaki T, Motohashi T, Tanida K, Okugawa T, Ikeda T. Torsion of a hydrosalpinx in a virgin patient with Mayer-Rokitansky-Küster-Hauser syndrome: report of a rare condition and its possible etiology. J Pediatr Adolesc Gynecol. 2013 Apr;26(2):e37-8.

[11] Lima M, Libri M, Aquino A, Gobbi D. Bilateral hydrosalpinx with asynchronous tubal torsion: an exceptional finding in a premenarcheal girl. J Pediatr Surg. 2011 Apr;46(4):e27-e29.

[12] Thakore SS, Chun MJ, Fitzpatrick K. Recurrent ovarian torsion due to paratubal cysts in an adolescent female. J Pediatr Adolesc Gynecol. 2012 Aug;25(4):e85-7.

[13] Pampal A, Atac GK, Nazli ZS, Ozen IO, Sipahi T. A rare cause of acute abdominal pain in adolescence: hydrosalpinx leading to isolated torsion of fallopian tube. J Pediatr Surg. 2012 Dec;47(12):e31-4.

[14] Masroor I, Khan N. Torsion of fallopian tube, fimbrial cyst. J Pak Med Assoc. 2008

[15] Zahiruddin S, Khan M, Iftikhar M. Fallopian Tube Cyst: A Rare Complication Of Tubal Sterilization. J Ayub Med Coll Abbottabad. 2016

[16] Macedo M, Kim B, Khoury R, Narkiewicz L. A rare case of right lower quadrant abdominal pain. Am J Emerg Med. 2017 Apr;35(4):668.e1-668.e2

[17] Murphy EM, Pereira N, Melnick AP, Spandorfer SD. Spontaneous bilateral torsion of fallopian tubes presenting as primary infertility. Womens Health (Lond). 2016 Jun;12(3):297-301

[18] Verpalen IM, de Boer J, van den Hoed RD, Schutte JM, Teklenburg G, Vossenkaul R. Geïsoleerde tubatorsie: zeldzaam of miskend? [Isolated fallopian tubal torsion; rare or overlooked?]. Ned Tijdschr Geneeskd. 2018 Jul 13;162

[19] Fadıloğlu E, Dur R, Demirda ̆̆ E, Öztürk Ç, Fadıloğlu Ş, Kaplan M, Tapısız ÖL. Isolated tubal torsion: Successful preoperative diagnosis of five cases using ultrasound and management with laparoscopy. Turk J 
Obstet Gynecol. 2017 Sep;14(3):187-190.

[20] Lourenco AP, Swenson D, Tubbs RJ, Lazarus E. Ovarian and tubal torsion: imaging findings on US, CT, and MRI. Emerg Radiol. 2014 Apr;21(2):179-87.

[ 21] Narayanan S, Bandarkar A, Bulas DI. Fallopian tube torsion in the pediatric age group: radiologic evaluation. J Ultrasound Med. 2014 Sep;33(9):1697-704.

[22] Sozen I, Kadako R, Fleischman S, Arici A. Diagnosis and laparoscopic management of a fallopian tube torsion following Irving tubal sterilization: a case report. Surg Endosc. 2002 Jan;16(1):217.

\section{Figure legends}

Fig.1. CT-image shows a cystic mass of $8 \mathrm{~cm}$ which is surrounding by oedematous stroma and engorged blood vessels

Fig.2. Intraoperative image of the ischemic left fallopian tube
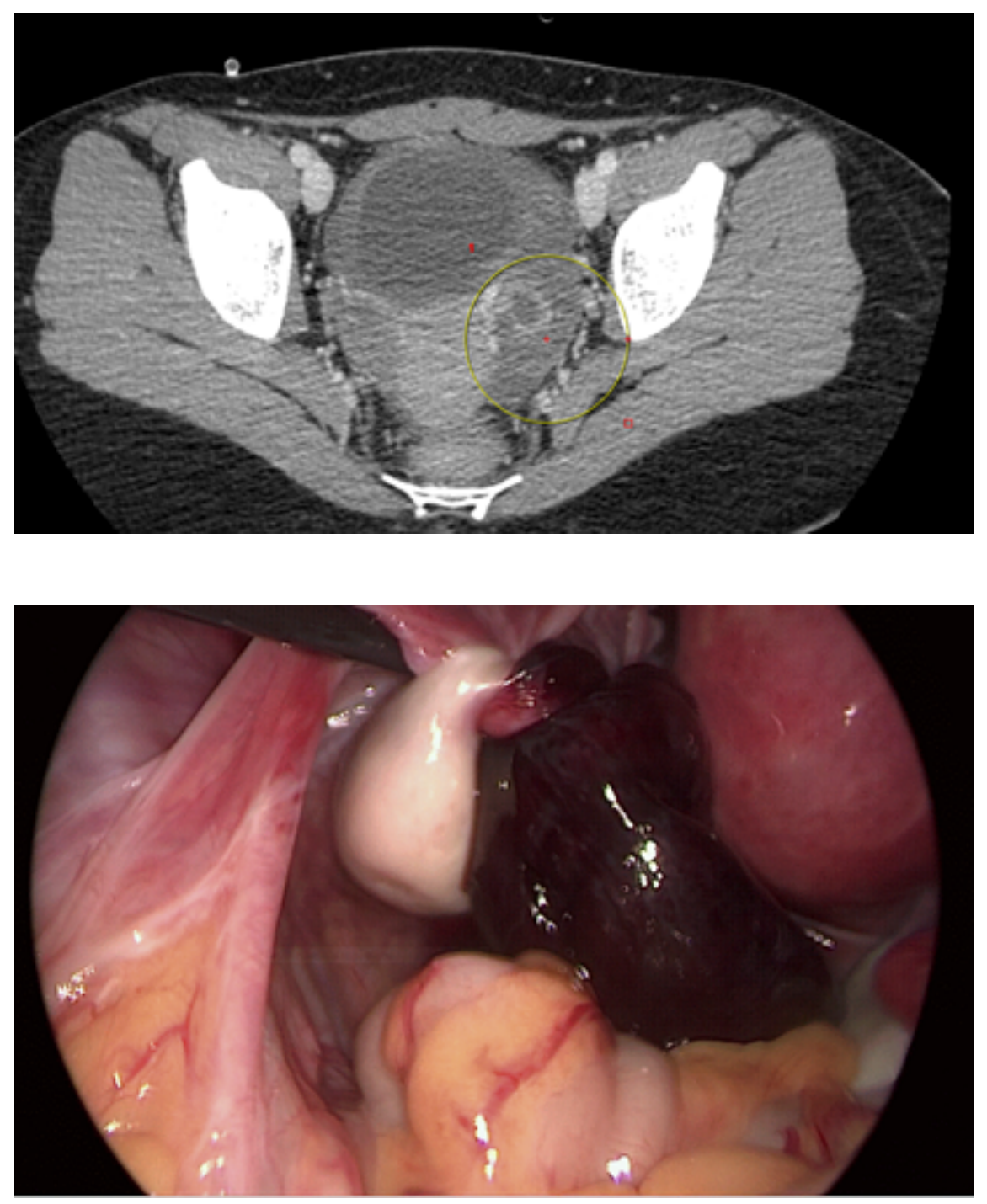